Revista Tecnologia e Ambiente, v. 21, 2014, Criciúma, Santa Catarina. ISSN: 1413-8131 (versão impressa) ISSN: 2358-9426 (versão eletrônica)

\title{
ESTRUTURA DA ASSEMBLEIA DE FORMIGAS (HYMENOPTERA: FORMICIDAE) EPIGÉICAS EM ÁREAS REABILITADAS APÓS MINERAÇÃO DE CARVÃO A CÉU ABERTO NO SUL DE SANTA CATARINA, BRASIL
}

\section{STRUCTURE OF EPIGEIC ANTS (HYMENOPTERA: FORMICIDAE) ASSEMBLY IN RECLAIMED AREAS AFTER SURFACE COAL MINING IN THE SOUTH OF SANTA CATARINA, BRAZIL}

\author{
João Luis Osório Rosado ${ }^{1}$ \\ Georg Henrique Beckmann ${ }^{2}$ \\ Robson Siqueiro Patricio ${ }^{3}$ \\ Birgit Harter-Marques ${ }^{4}$
}

\begin{abstract}
Resumo
A mineração de carvão representa uma das atividades que causa elevada degradação ambiental, com grande destaque para a região sul de Santa Catarina. O processo de reabilitação das áreas mineradas passa por uma série de procedimentos, visando à restauração das condições mínimas necessárias para o reestabelecimento da biodiversidade. Pesquisas que avaliem este processo são fundamentais para $o$ monitoramento do grau de reestruturação destas áreas. $\mathrm{O}$ objetivo do presente estudo foi avaliar a estrutura da assembleia de formigas em quatro áreas reabilitadas após mineração de carvão a céu aberto, comparando-se os dados com uma área de mata nativa, tomada como testemunha das condições originais na região sul do estado de Santa Catarina. A amostragem foi realizada mensalmente nas cinco áreas durante 12 meses, utilizando-se 10 armadilhas do tipo pitfall, por local de coleta. Um total de 50 espécies foi coletado, distribuídas em 20 gêneros e oito subfamílias. As análises evidenciaram que as áreas reabilitadas apresentaram baixa riqueza de espécies de formigas, além de uma composição significativamente distinta daquela observada na área do remanescente. Destaca-se, contudo que o presente estudo provém o primeiro inventário da mirmecofauna para as regiões reabilitadas, sendo necessária a continuação de estudos que incluem áreas sob condições mais avançadas de regeneração.
\end{abstract}

Palavras-chave: mirmecofauna, restauração de áreas impactadas, bioindicadores, Mata Atlântica.

\section{Abstract}

Coal mining represents one of the activities that cause environmental degradation, with great emphasis on the southern region of Santa Catarina. The reclaim process of the mined areas undergoes a series of procedures which aim the restoration of the minimum

\footnotetext{
1 Laboratório de Interação Animal-Planta, Universidade do Extremo Sul Catarinense (UNESC), Criciúma, Santa Catarina.

${ }^{2}$ Ex-Bolsista do Programa de Iniciação Científica (PIBIC), Universidade do Extremo Sul Catarinense (UNESC), Criciúma, Santa Catarina.

3 Laboratório de Interação Animal-Planta, Universidade do Extremo Sul Catarinense (UNESC), Criciúma, Santa Catarina.

${ }^{4}$ Docente do Programa de Pós-Graduação em Ciências Ambientais (PPGCA), Universidade do Extremo Sul Catarinense (UNESC), Av. Universitária, Bairro Universitário, CEP 88806-000, Criciúma, Santa Catarina. E-mail: bhm@unesc.net
} 
Revista Tecnologia e Ambiente, v. 21, 2014, Criciúma, Santa Catarina. ISSN: 1413-8131 (versão impressa) ISSN: 2358-9426 (versão eletrônica)

conditions necessary for biodiversity reestablishment. Researches which evaluate this process are important for monitoring the restructuring degree of these areas. The aim of this study was to evaluate the structure of the ant species assembly in four reclaimed areas after surface coal mining, comparing the data with a native forest area taken as control of the original conditions in southern of Santa Catarina. Sampling was conducted monthly in the five areas for 12 months using 10 pitfall traps in each sample area. A total of 50 species were collected, distributed in 20 genera and eight subfamilies. Data analysis showed that the reclaimed areas presented low species richness of ants, and a significantly different composition from that observed in the native area. We highlighted, however, that this study represents the first inventory of the ant fauna realized in reclaimed areas, and further studies which include areas under more advanced regeneration conditions are required.

Keywords: antfauna, restoration of impacted areas, bioindicators, Atlantic rainforest.

\section{Introdução}

A exploração intensiva dos recursos naturais pelo homem e o consequente impacto sobre os habitats tem alterado a maior parte dos ecossistemas terrestres (QUEIROZ et al., 2006). Dentre as atividades antrópicas que mais interferem e degradam o meio ambiente, destaca-se a extração de carvão mineral. O carvão mineral é o combustível fóssil com maior disponibilidade no mundo e, desse modo, de grande importância para o setor energético mundial. Apesar de sua importância econômica, a exploração, o beneficiamento e a utilização desse recurso representam atividades com elevado impacto ambiental através da contaminação do solo, do ar e dos recursos hídricos, alterando a paisagem e afetando significativamente a biodiversidade (SANCHEZ; FORMOSO, 1990; DIAS; GRIFFITH, 1998; COSTA; ZOCHE, 2009).

A extração do carvão mineral pode se dar através de minas subterrâneas ou a céu aberto, sendo a opção por uma ou por outra modalidade depende, basicamente, da profundidade e do tipo de solo o qual o minério se encontra (ANEEL, 2008). No processo de mineração a céu aberto, a remoção das camadas é feita de forma desordenada, onde os horizontes do solo são descaracterizados pela inversão de suas camadas, gerando pilhas de materiais geológicos que têm na sua base a camada fértil do solo (DE LUCA; GASTALDON, 1999), causando, consequentemente, perda de solo fértil, supressão da presença de animais e plantas, além de destruição do banco de sementes (CITADINI-ZANETTE et al., 2009; KLEIN et al., 2009).

Devido ao impacto causado, a legislação ambiental brasileira impõe às empresas mineradoras a necessidade de realizar a reabilitação das áreas degradadas após a 
Revista Tecnologia e Ambiente, v. 21, 2014, Criciúma, Santa Catarina. ISSN: 1413-8131 (versão impressa) ISSN: 2358-9426 (versão eletrônica)

exploração. O processo de recuperação das áreas de mineração a céu aberto, por exemplo, passa pela recomposição topográfica e paisagística, assim como pela reconstrução do solo através da utilização dos solos naturais e materiais litológicos sobrejacentes às camadas de carvão. Este procedimento visa reconstituir a qualidade e a capacidade produtiva original do solo, onde deverá ser novamente estabelecida uma vegetação de cobertura com a reintrodução de espécies vegetais herbáceas e arbóreas, nativas e/ou exóticas (PINTO, 1997; CAMPOS et al., 2010) e, consequentemente, a recolonização pela fauna, principalmente de invertebrados.

Embora não existam protocolos precisos na reconstrução de áreas mineradas, pesquisas são fundamentais para buscar alternativas para um melhor processo de recuperação de áreas reconstruídas e para o monitoramento do grau de reestruturação destas áreas. Deste modo, as comunidades de insetos edáficos e/ou processos bioquímicos mediados por eles são indicadores potencialmente sensíveis às mudanças na qualidade do solo e indispensáveis em programas de monitoramento dos processos de recuperação ambiental de áreas degradadas (JORDAN et al., 1995). Peck et al. (1998) destacam que os insetos são considerados bons bioindicadores, pois respondem rapidamente ao estresse ambiental, tem curtos períodos de geração e, em geral são facilmente amostrados e identificados.

Dentre os insetos, as formigas estão entre os grupos de organismos mais comumente utilizados como bioindicadores (e.g. MAJER, 1983; ANDERSEN et al., 2004; OTTONETTI et al., 2006; MAJER et al., 2007; PHILPOTT et al., 2010; RIBAS et al., 2012a). Seu uso tem sido proposto para o monitoramento de recuperação de impactos ambientais e como indicadores de conservação, pois são largamente distribuídas; com uma riqueza relativamente alta de espécies; são extremamente abundantes; ocupam níveis tróficos elevados, exercendo papel significante no funcionamento dos ecossistemas; possuem taxa especializados; são facilmente amostradas e separadas em morfo-espécies; com taxonomia e ecologia bem conhecidas; além de serem sensíveis às mudanças nas condições ambientais (MAJER, 1983; AGOSTI et al., 2000; UNDERWOOD; FISCHER, 2006; RIBAS et al., 2012a). Tais características podem fornecer inferências confiáveis sobre as implicações ecológicas e funcionais dos distúrbios (RIBAS et al., 2012a). Além disso, as formigas podem prover, até certo ponto, uma indicação da abundância e diversidade de outros taxa de 
Revista Tecnologia e Ambiente, v. 21, 2014, Criciúma, Santa Catarina. ISSN: 1413-8131 (versão impressa) ISSN: 2358-9426 (versão eletrônica)

invertebrados, característica esta importante, tendo-se em vista que inventariamentos completos de invertebrados são onerosos e demandam muito tempo, tornando-se, na maioria das vezes, impraticáveis (LINDENMAYER, 1999).

A diversidade, abundância e a composição de espécies de formigas são frequentemente relacionadas com a variedade de locais de nidificação e alimentação, condições (micro)climáticas, e as interações competitivas entre as espécies (HÖLLDOBLER; WILSON, 1990). Deste modo, alterações antropogênicas na paisagem podem ser capazes de causar mudanças nas condições microclimáticas para nidificação e forrageamento das formigas (LOBRY DE BRUYN, 1999), além da redução na disponibilidade de alimentos e aumento na competição interespecífica (HÖLLDOBLER; WILSON, 1990).

No Brasil, o uso de formigas como indicadores tem aumentado significativamente a cada ano (RIBAS et al., 2012a), sendo utilizadas para avaliar diferentes tipos de impactos, tais como o fogo, desmatamento e fragmentação, florestamentos comerciais, diferentes usos do solo e práticas em agroecossistemas. Cabe destacar, contudo, que poucos estudos têm avaliado a recuperação de áreas mineradas (e.g. PEREIRA et al., 2007; RIBAS et al., 2012b e c). Na maioria dos estudos, os autores analisaram a resposta da riqueza e composição de espécies de formigas, obtendo resultados contrastantes. Alguns estudos encontraram respostas significantes da riqueza de espécies de formigas a diferentes impactos ambientais (COSTA et al., 2010; DELABIE et al., 2006; PEREIRA et al., 2007; SILVA; BRANDÃO, 1999; RIBAS et al., 2012c), enquanto outros concluíram que mudanças na composição de espécies seria um parâmetro mais adequado para a bioindicação (COELHO et al., 2009; SCHIMDT; DIEHL, 2008; VASCONCELOS et al., 2000), especialmente na dinâmica dos ambientes (GOLLAN et al., 2011). Para Ribas et al. (2012c), a riqueza de espécies pode ser um parâmetro melhor para bioindicação de distúrbio em áreas homogêneas, do que em relação a áreas dinâmicas e heterogêneas onde a composição de espécies pode ser mais apropriada.

Logo, este estudo teve como objetivo avaliar a estrutura da assembleia de formigas epigéicas em áreas reabilitadas após mineração de carvão a céu aberto, comparando-se os dados com uma área de remanescente de mata nativa adjacente em estágio avançado de regeneração, tomada como testemunha das condições ambientais 
Revista Tecnologia e Ambiente, v. 21, 2014, Criciúma, Santa Catarina. ISSN: 1413-8131 (versão impressa) ISSN: 2358-9426 (versão eletrônica)

originais na região Sul do Estado de Santa Catarina. Destaca-se também, que este estudo representa o primeiro passo que poderá servir como alicerce para o estabelecimento de programas de monitoramento das áreas de recuperação após mineração de carvão, utilizando-se as formigas como organismos bioindicadores.

\section{Materiais e Métodos}

\section{Áreas de estudo}

As áreas de estudo estão inseridas no Bioma Mata Atlântica, que caracteriza-se pela presença de diversos ecossistemas, incluindo desde formações florestais (Floresta Ombrófila Densa, Ombrófila Mista, Estacional Semidecidual, Estacional Decidual e Ombrófila Aberta) até ecossistemas associados com restingas, manguezais e campos de altitude (MMA, 2011). A região de estudo está localizada dentro da bacia carbonífera catarinense, que se constitui por uma faixa de aproximadamente $100 \mathrm{~km}$ de comprimento e largura média de $20 \mathrm{~km}$, entre a Serra Geral a oeste e o maciço granítico da Serra do Mar a leste, onde se desenvolveram importantes centros de mineração de carvão, especialmente nos municípios de Criciúma, Araranguá, Lauro Müller, Siderópolis e Urussanga (BELOLLI et al., 2002).

$\mathrm{O}$ estudo foi realizado em cinco áreas próximas entre si, pertencentes à Companhia Siderúrgica Nacional (CSN), localizadas no município de Siderópolis, no sul do Estado de Santa Catarina (Fig. 1).

Figura 1. Localização das áreas de estudo na região Sul do Estado de Santa Catarina, Brasil. A1 a A4 - áreas reabilitadas; A5 - área de remanescente de Floresta Ombrófila Densa.

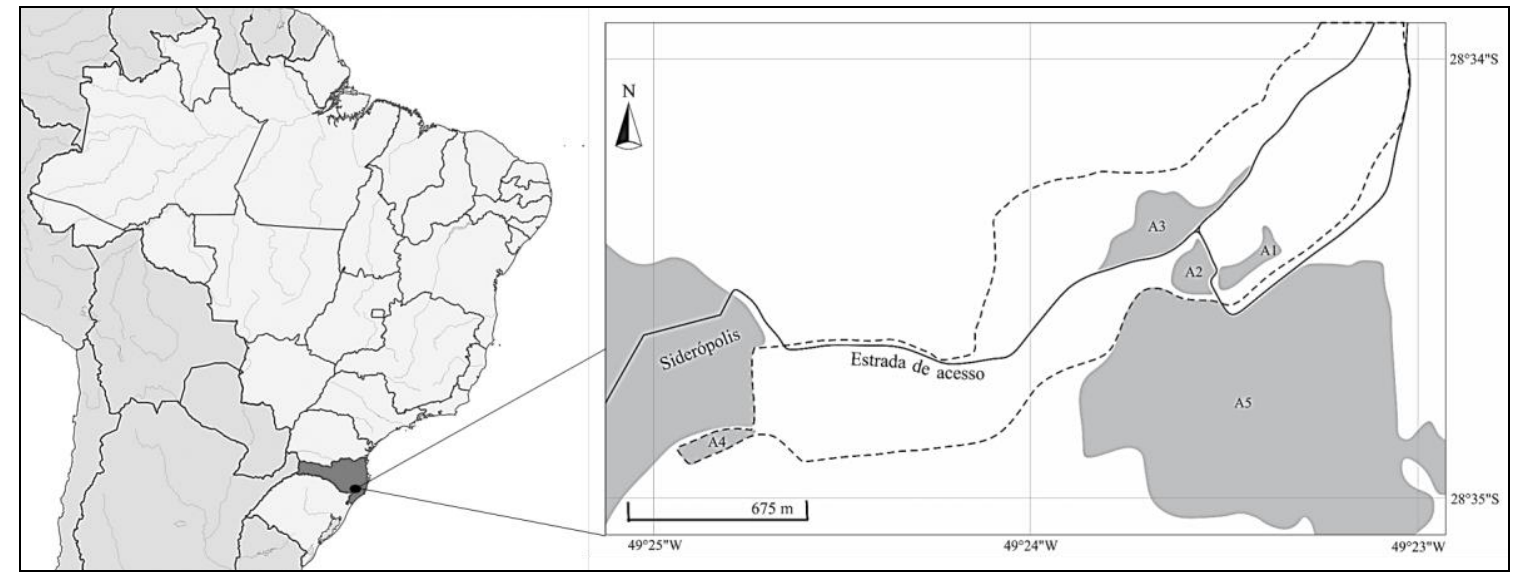

Fonte: adaptado Wikipedia e Google Earth 
Revista Tecnologia e Ambiente, v. 21, 2014, Criciúma, Santa Catarina. ISSN: 1413-8131 (versão impressa) ISSN: 2358-9426 (versão eletrônica)

As áreas A1, A2, A3 e A4 caracterizam-se como locais que sofreram atividade de mineração de carvão a céu aberto, e que passaram por um processo de reabilitação no período de 2002 a 2006. Para o processo de recuperação do solo em todas as áreas foram utilizadas camadas de argila e turfa. Seguem demais especificidades de cada área: Área 1 - (28 34'S 49²4'W). Reabilitação concluída em 2006. Vegetação predominante durante o período do estudo: capim-gordura (Melinis minutiflora P. Beauv.);

Área 2 - (28 34'S 49²4'W). Reabilitação concluída em 2006. Vegetação predominante durante o período do estudo: capim-gordura (M. minutiflora); bracatinga (Mimosa scabrella Benth.); maricá (Mimosa bimucronata (DC.) Kuntze);

Área 3 - (28 $46^{\prime}$ 'S 49²4'W). Reabilitação concluída em 2004. Vegetação predominante durante o período do estudo: capim-gordura (M. minutiflora); bracatinga (M. scabrella); maricá (M. bimucronata). Área bastante úmida (antiga lagoa de mineração que foi aterrada) com vegetação pouco desenvolvida, apresentando alguma vegetação arbustiva espontânea, constituída basicamente por Baccharis spp.;

Área 4 - (28 $35^{\prime}$ S $\left.49^{\circ} 25^{\prime} \mathrm{W}\right)$. Reabilitação concluída em 2004. Vegetação predominante durante o período do estudo: capim-gordura (M. minutiflora); bracatinga (M. scabrella). No momento da coleta a vegetação de bracatinga apresentava-se mais desenvolvida em relação às outras áreas ( $\cong 6 \mathrm{~m}$ de altura), exibindo quantidade significativa de serapilheira;

Área 5 - $\left(28^{\circ} 34^{\prime} S 4^{\circ} 24^{\prime} \mathrm{W}\right)$. Caracteriza-se como uma área de remanescente de Floresta Ombrófila Densa, secundária e em estádio avançado de regeneração natural.

De acordo com a classificação de Köppen (1948), o clima da região sul de Santa Catarina é do tipo subtropical úmido (Cfa), sem estação seca definida e com verões quentes, com precipitação anual variando de $1.400 \mathrm{~mm}$ a $1.800 \mathrm{~mm}$ e temperatura média de $19^{\circ} \mathrm{C}$. No inverno ocorrem gradientes térmicos inferiores a $10^{\circ} \mathrm{C}$, permitindo a ocorrência de geadas (IPAT, 2000).

\section{Coleta de dados}

A amostragem foi realizada mensalmente nas cinco áreas, durante o período de março de 2007 a fevereiro de 2008, totalizando-se 12 coletas em cada área. Para cada coleta foram utilizadas 10 armadilhas do tipo "pitfall" dispostas a cada 10m em um transecto linear de $100 \mathrm{~m}$, e respeitando-se o limite de $20 \mathrm{~m}$ das bordas. Os pitfalls 
Revista Tecnologia e Ambiente, v. 21, 2014, Criciúma, Santa Catarina. ISSN: 1413-8131 (versão impressa) ISSN: 2358-9426 (versão eletrônica)

utilizados consistiam de recipientes plásticos de 400ml, contendo uma solução de água e detergente neutro a 5\%, sendo enterrados até sua borda superior de forma a permanecerem nivelados com o solo. Em cada coleta os pitfalls foram deixados no campo por $72 \mathrm{hs}$, sendo posteriormente, o material obtido armazenado em recipientes com álcool 70\% e transportados para o Laboratório de Interação Animal-Planta (LIAP) da Universidade do Extremo Sul Catarinense (UNESC). Logo após, o material foi triado e identificado ao nível de gênero com uso da chave dicotômica de Palacio e Fernández (2003). Quando possível, a identificação foi feita em nível de espécie, caso contrário os grupos permaneceram como morfo-espécies. A determinação das espécies foi realizada através da comparação com o material disponível na coleção do Museu de Zoologia da Universidade de São Paulo (MZSP). Exemplares de todas as espécies coletadas foram depositados nas coleções do MZSP e do LIAP.

\section{RESULTADOS}

No total foram amostradas 50 morfo-espécies (50\% identificadas a nível específico), distribuídas em 20 gêneros e oito subfamílias. Myrmicinae foi a subfamília com o maior número de espécies (24 espécies), seguido por Formicinae (dez espécies), Ponerinae (cinco espécies), Dolichoderinae (quatro espécies), Dorylinae, Ectatomminae e Pseudomyrmecinae (cada uma com duas espécies), e Heteroponerinae (uma espécie). Os três gêneros com maior número de espécies foram Pheidole Westwood, 1839 (13 espécies), Camponotus Mayr, 1861 (oito espécies), e Linepithema Mayr, 1866 (quatro espécies) (Tabela 1).

Tabela 1. Mirmecofauna epigéica coletada com armadilhas pitfall em área reabilitada após mineração de carvão a céu-aberto (A1 a A4) e uma área de remanescente de mata nativa (A5) na região Sul do Estado de Santa Catarina, no período de março de 2007 a fevereiro de 2008.

\begin{tabular}{|c|c|c|c|c|c|}
\hline Identificações & $\overline{A 1}$ & $\overline{A 2}$ & $\mathbf{A 3}$ & $\mathbf{A 4}$ & A5 \\
\hline \multicolumn{6}{|l|}{ Dolichoderinae } \\
\hline Linepithema humile (Mayr, 1868) & & & & $X$ & \\
\hline Linepithema micans (Forel, 1908) & & & & $\mathrm{X}$ & \\
\hline Linepithema pulex Wild, 2007 & & & & $X$ & \\
\hline
\end{tabular}


Revista Tecnologia e Ambiente, v. 21, 2014, Criciúma, Santa Catarina. ISSN: 1413-8131 (versão impressa) ISSN: 2358-9426 (versão eletrônica)

\begin{tabular}{|c|c|c|c|c|c|}
\hline Identificações & $\overline{A 1}$ & $\overline{A 2}$ & $\overline{\mathrm{A3}}$ & $\overline{\mathrm{A4}}$ & $\overline{\mathrm{A5}}$ \\
\hline Linepithema sp.1 & & & & $\mathrm{X}$ & \\
\hline \multicolumn{6}{|l|}{ Dorylinae } \\
\hline Eciton burchellii (Westood, 1842) & & & & & $\mathrm{X}$ \\
\hline Eciton quadriglume (Haliday, 1836) & & & & & $\mathrm{X}$ \\
\hline
\end{tabular}

\section{Ectatomminae}

Gnamptogenys striatula Mayr, $1884 \quad \mathrm{X}$

Ectatomma edentatum (Roger, 1863) X X

\section{Formicinae}

Camponotus atriceps (Smith, 1858)

X $\quad X \quad X \quad X$

Camponotus fuscocinctus Emery, 1888

Camponotus lespesii Forel, 1886

Camponotus melanoticus Emery, 1894

Camponotus mus Roger, 1863

Camponotus rufipes (Fabricius, 1775)

Camponotus sp.1

$\begin{array}{ccccc} & & & X \\ & & & X & X \\ & X & X & X & \\ X & & & X & \\ & & & X & \\ & & & X\end{array}$

Camponotus sp. 6

$\mathrm{X}$

Nylanderia fulva (Mayr, 1862)

Nylanderia sp.3

$\begin{array}{llll}\text { X } & \text { X } & \text { X } & \text { X } \\ & \text { X } & & \end{array}$

\section{Heteroponerinae}

Heteroponera dentinodis (Mayr, 1887)

$\mathrm{X}$

\section{Myrmecinae}

Acromyrmex striatus (Roger, 1863)

Acromyrmex rugosus (Smith, 1858)

Cephalotes pusillus (Klug, 1824)

Crematogaster bingo Forel, 1908

$\begin{array}{ll}X & X \\ X\end{array}$

Crematogaster pr. torosa

$\mathrm{X}$

Crematogaster sp. 3

X

Cyphomyrmex (gr. rimosus) sp.1

X X

Pheidole (gr. diligens) pr. angusta

Pheidole (gr. biconstricta) sp.1

X $\quad$ X $\quad$ X 
Revista Tecnologia e Ambiente, v. 21, 2014, Criciúma, Santa Catarina. ISSN: 1413-8131 (versão impressa) ISSN: 2358-9426 (versão eletrônica)

\begin{tabular}{|c|c|c|c|c|c|}
\hline Identificações & $\mathbf{A 1}$ & $\overline{A 2}$ & $\overline{A 3}$ & $\mathbf{A 4}$ & $\overline{\mathrm{A5}}$ \\
\hline Pheidole sp.3 & & $\mathrm{X}$ & $\mathrm{X}$ & $\bar{X}$ & $\mathrm{X}$ \\
\hline Pheidole sp.4 & & & & & $X$ \\
\hline Pheidole sp.5 & & & & & $\mathrm{X}$ \\
\hline Pheidole sp.11 & & & & & $X$ \\
\hline Pheidole sp.13 & & & & & $X$ \\
\hline Pheidole sp.14 & & & & & $X$ \\
\hline Pheidole sp.15 & & & & & $\mathrm{X}$ \\
\hline Pheidole sp.16 & & & & & $\mathrm{X}$ \\
\hline Pheidole (gr. fallax) sp.17 & & & & & $\mathrm{X}$ \\
\hline Pheidole sp.18 & & & & & $\mathrm{X}$ \\
\hline Pheidole sp.19 & & & & & $\mathrm{X}$ \\
\hline Pogonomyrmex naegelli (Emery, 1878) & & $\mathrm{X}$ & & & \\
\hline Solenopsis geminata (Fabricius, 1804) & $\mathrm{X}$ & $\mathrm{X}$ & & $X$ & \\
\hline Solenopsis sp.2 & $X$ & $\mathrm{X}$ & $\mathrm{X}$ & & \\
\hline Wasmannia pr. sulcaticeps & & & $\mathrm{X}$ & & $\mathrm{X}$ \\
\hline \multicolumn{6}{|l|}{ Ponerinae } \\
\hline Hypoponera sp.1 & $\mathrm{X}$ & $\mathrm{X}$ & $\mathrm{X}$ & $\mathrm{X}$ & $\mathrm{X}$ \\
\hline Neoponera crenata (Roger, 1861) & & & & & $\mathrm{X}$ \\
\hline Odontomachus meinerti Forel, 1905 & & & & & $\mathrm{X}$ \\
\hline Odontomachus chelifer (Latreille, 1802) & & & & & $\mathrm{X}$ \\
\hline Pachycondyla striata (Smith, 1858) & $\mathrm{X}$ & $\mathrm{X}$ & $\mathrm{X}$ & $\mathrm{X}$ & $\mathrm{X}$ \\
\hline \multicolumn{6}{|l|}{ Pseudomyrmecinae } \\
\hline Pseudomyrmex (gr. aculatus) sp.1 & & & & & $\mathrm{X}$ \\
\hline Pseudomyrmex pr. urbanus & & & & & $\mathrm{X}$ \\
\hline Número total de espécies & 9 & 13 & 11 & 18 & 33 \\
\hline
\end{tabular}

No presente estudo, a área do remanescente (A5) apresentou a maior riqueza de espécies observada $\left(\mathrm{S}_{\mathrm{obs}}=33\right)$, quando comparado ao número médio de espécies nas áreas reabilitadas $\left(\mathrm{S}_{\mathrm{obs}}=12,75\right)$. Entre as áreas de reabilitação, a área $\mathrm{A} 1$ obteve menor número de espécies $\left(S_{\mathrm{obs}}=9\right)$, seguido pela área $\mathrm{A} 3\left(\mathrm{~S}_{\mathrm{obs}}=11\right), \mathrm{A} 2\left(\mathrm{~S}_{\mathrm{obs}}=13\right)$ e $\mathrm{A} 4\left(\mathrm{~S}_{\mathrm{obs}}\right.$ =18). Do total de espécies, somente sete foram compartilhadas pelos dois ambientes 
Revista Tecnologia e Ambiente, v. 21, 2014, Criciúma, Santa Catarina. ISSN: 1413-8131 (versão impressa) ISSN: 2358-9426 (versão eletrônica)

(reabilitada x remanescente), enquanto 26 espécies ocorreram exclusivamente no remanescente de mata nativa e 17 espécies foram exclusivas das áreas reabilitadas. Acromyrmex rugosus (Smith, 1858), Camponotus atriceps (Smith, 1858), C. melanoticus Emery, 1894, C. mus Roger, 1863, C. rufipes (Fabricius, 1775), Camponotus sp.1, Cyphomyrmex sp.1, Linepithema humile (Mayr, 1868), L. micans (Forel, 1908), L. pulex Wild, 2007, Linepithema sp.1, Nylanderia fulva (Mayr, 1862), Nylanderia sp.3, Pheidole sp.1, Pogonomyrmex naegelli (Emery, 1878), Solenopsis geminata (Fabricius, 1804) e Solenopsis sp.2 foram as espécies que ocorreram exclusivamente na área reabilitada. Acromyrmex striatus (Roger, 1863), Camponotus lespesii Forel, 1886, Cephalotes pusillus (Klug, 1824), Hypoponera sp.1, Pachycondyla striata (Smith, 1858), Pheidole sp.3 e Wasmannia pr. sucalticeps foram as espécies compartilhadas entre os dois ambientes.

Com relação aos grupos funcionais apresentados por Brandão et al. (2012), a área de remanescente apresentou exemplares pertencentes aos grupos das espécies generalistas e/ou arbóreas dominantes (gêneros Camponotus, Crematogaster, Pheidole e Wasmannia) com 16 espécies, além de espécies predadoras generalistas (Ectatomma, Gnamptogenys, Heteroponera, Hypoponera, Neoponera, Odontomachus e Pachycondyla) $(\mathrm{S}=8)$, predadoras arbóreas (Pseudomyrmex) $(\mathrm{S}=2)$, cultivadoras de fungo sobre material vegetal fresco (Acromyrmex) $(\mathrm{S}=1)$, legionárias (Eciton) $(\mathrm{S}=2)$ e arbóreas que se alimentam de pólen (Cephalotes) $(\mathrm{S}=1)$. Na área reabilitada foi coletado um mesmo número de espécies generalistas (gêneros Camponotus, Linepithema, Nylanderia, Solenopsis, Pheidole e Wasmannia) $(\mathrm{S}=16)$, de cultivadoras de fungo sobre material vegetal fresco (Acromyrmex) $(\mathrm{S}=1)$ e de arbóreas que se alimentam de pólen (Cephalotes) $(\mathrm{S}=1)$, mas um menor número de espécies predadoras generalistas (Hypoponera sp. e Pachycondyla) $(\mathrm{S}=2)$. No entanto, na área reabilitada também foram encontrados dois grupos que não haviam sido coletados na área do remanescente, são eles: coletora de sementes do gênero Pogonomyrmex, e outra espécie pertencente ao grupo das cultivadoras de fungo sobre material em decomposição do gênero Cyphomyrmex.

\section{DISCUSSÃO}


Revista Tecnologia e Ambiente, v. 21, 2014, Criciúma, Santa Catarina. ISSN: 1413-8131 (versão impressa) ISSN: 2358-9426 (versão eletrônica)

No presente estudo a predominância das subfamílias Myrmicinae, Formicinae e Ponerinae, e dos gêneros Pheidole e Camponotus, deve-se ao fato de serem muito comuns e abundantes, especialmente na região Neotropical. Esta constatação corrobora a maioria dos estudos realizados no Brasil, independentemente da metodologia e do local de estudo (e.g. MARINHO et al., 2002; CÔRREA et al., 2006; ANDRADE et al., 2007; ROSADO et al., 2012). A maior representatividade desses grupos deve-se, possivelmente, a presença de muitas espécies agressivas e dominantes, generalistas e/ou onívoras (SILVESTRE et al., 2003).

Entre os gêneros predominantes, Pheidole é considerado um grupo hiperdiverso, amplamente distribuído, generalista e conhecido pelo seu sistema de recrutamento em massa, que permite dominar recursos alimentares e excluir de maneira eficiente seus competidores (FOWLER, 1993; WILSON, 2003). Já as espécies de Camponotus apresentam ampla distribuição geográfica, sendo constituído por espécies generalistas, de hábitos terrícolas e arborícolas dominantes, segundo as guildas descritas para o Cerrado (SILVESTRE et al., 2003; DELABIE et al., 2006) e para a Mata Atlântica (BRANDÃO et al., 2012). Este gênero possui muitas espécies com elevada capacidade de invasão a novos ambientes, em decorrência tanto de sua adaptabilidade, quanto da flexibilidade de sua dieta (RAMOS et al., 2003). Outras espécies oportunistas como aquelas pertencentes ao gênero Linepithema preferem habitats mais abertos (UNDERWOOD; FISHER, 2006), aumentando sua ocorrência em ambientes degradados.

A baixa riqueza de espécies presente nas áreas reabilitadas deve-se, possivelmente, à menor heterogeneidade ambiental, com a consequente redução da variedade de substratos para a alimentação e nidificação, restringindo assim a capacidade de suporte do ambiente (HÖLLDOBLER; WILSON, 1990; LOBRY DE BRUYN, 1999; MARINHO et al., 2002). A estrutura da vegetação, tal como o número de espécies vegetais, afeta a comunidade de formigas (LASSAU; HOCHULI, 2004; VASCONCELOS et al., 2008, 2010), e a redução na disponibilidade de recursos e/ou a qualidade destes recursos é relacionado a redução na riqueza de espécies (RIBAS et al., 2003). Para Pereira et al. (2007), ambientes degradados ou com baixa diversificação vegetal apresentam limitações à presença de organismos, devido à falta de recursos proporcionados por estes ambientes, consequentemente reduzindo a diversidade de 
Revista Tecnologia e Ambiente, v. 21, 2014, Criciúma, Santa Catarina. ISSN: 1413-8131 (versão impressa) ISSN: 2358-9426 (versão eletrônica)

espécies e de grupos funcionais. A exemplo, a reduzida densidade e riqueza de espécies de árvores na área reabilitada pode ter sido fator determinante para a ausência de espécies pertencentes aos gêneros Crematogaster e Pseudomyrmex, tipicamente com hábitos arborícolas (BRANDÃO et al., 2012). A presença de um solo construído na área reabilitada, possivelmente, é outro fator importante na seleção das espécies que irão se estabelecer neste ambiente. A camada de argila utilizada na recuperação das áreas, além de reduzir a permeabilidade e aeração do solo (ROCHA-NICOLEITE et al., 2013), pode representar um importante dificultador para a construção dos ninhos subterrâneos.

A redução da heterogeneidade é também destacada como um fator determinante para o aumento da competição interespecífica (HÖLLDOBLER; WILSON, 1990; CORREA et al., 2006; LOPES et al., 2010). Logo, para o presente estudo, a competição é maior na área de reabilitação pelo fato que essas áreas apresentam baixa quantidade e qualidade de recursos, o que dificulta o processo de recolonização por espécies subordinadas e, consequentemente, impede a recuperação das áreas para uma condição próxima das áreas nativas. A presença de espécies exclusivas nas áreas reabilitadas pode estar relacionada à migração de espécies oportunistas provindas de outras matrizes adjacentes, com composição distinta daquela observada na área do remanescente, podendo influenciar significativamente o processo de recolonização nas áreas reabilitadas, devido à competição interespecífica. Este fator deve explicar a maior riqueza observada na área $\mathrm{A} 4$ em relação às demais áreas reabilitadas, pois a mesma encontra-se mais próxima ao ambiente urbano. Espécies pertencentes aos gêneros Camponotus, Linepithema, Nylanderia e Solenopsis são frequentemente citadas em estudos realizados em ambientes urbanos (CHACÓN DE ULLOA, 2003; LUTINSKI et al., 2013). As formigas urbanas possuem uma série de características biológicas e ecológicas que favorecem sua dispersão nos mais diversos locais (HÖLLDOBLER; WILSON, 1990; HOLWAY et al., 2002; TSUTSUI; SUAREZ, 2003), como: estrutura social polígena (colônias com muitas rainhas funcionais, formando sociedades unicoloniais, nas quais, não há limites bem definidos e suas colônias se multiplicam por fragmentação (Budding) (HÖLLDOBLER; WILSON, 1990); não apresentam agressão intraespecífica entre seus ninhos, mas são muito agressivas frente a outras espécies (PASSERA, 1993, 1994); pequena demanda espacial para a construção dos ninhos; e 
Revista Tecnologia e Ambiente, v. 21, 2014, Criciúma, Santa Catarina. ISSN: 1413-8131 (versão impressa) ISSN: 2358-9426 (versão eletrônica)

recrutamento massivo que permite mobilizar operárias rapidamente para explorar uma fonte de alimento ou excluir potenciais competidores (FOWLER et al., 1994).

Além disso, muitas das espécies exclusivas das áreas reabilitadas possuem hábito mais generalista em relação às condições do ambiente. As espécies do gênero Solenopsis, por exemplo, são caracteristicamente generalistas no que se refere ao habitat e à dieta (GONÇALVES; NUNES, 1984), destacando-se por sua agressividade na utilização de recursos do solo e serapilheira, sendo frequentes, tanto em ambientes nativos, como alterados (DELABIE; FOWLER, 1995). As formigas deste grupo são capazes de suportar longos períodos de escassez de alimentos, além de possuírem eficientes estratégias de recrutamento em massa (FOWLER et al., 1991). Vasconcelos (1999) verificou, por exemplo, que Solenopsis geminata aumentou sua abundância em áreas abertas. Esta espécie é considerada chave em algumas áreas agrícolas, devido aos seus efeitos na estrutura da comunidade de plantas e de artrópodes (RISCH; CARROLL, 1982; CARROL; RISCH, 1984) e, sendo assim, pode ter um papel crucial nos processos de regeneração florestal nestas áreas (VASCONCELOS, 1999).

Destaca-se também a presença das espécies Cyphomyrmex sp.1 e Pogonomyrmex naegelii, também coletadas exclusivamente nas áreas reabilitadas, apesar de representarem grupos com hábito relativamente mais especializado. Contudo, a primeira espécie, pertencente à tribo Attini, é especializada no cultivo de fungos através de uma ampla variedade de substratos, como folhas (raramente frescas, mas principalmente já deterioradas), flores, frutos, sementes, fezes, liquens, musgos e carcaças de outros artrópodes (LEAL; OLIVEIRA, 1998), hábitos estes, que permitiram seu estabelecimento em duas das áreas reabilitadas. Já Pogonomyrmex naegelii caracteriza-se pela preferência por nidificar em zonas abertas e mais secas (FERNÁNDEZ, 2003), e em solos descobertos como observado por Belchior et al. (2012) em uma área de Savana no Cerrado, habitada por vegetação lenhosa dispersa e com poucas espécies herbáceas. Segundo estes autores, P. naegelii possui uma dieta generalista, compreendendo muitas espécies de sementes (preferencialmente de gramíneas) e de artrópodes (principalmente formigas e térmitas), além de fragmentos de material vegetal e animal. Logo, tais características tornam esta espécie como potencial colonizadora das áreas reabilitadas, apesar de ter sido observada somente na área A2. 
Revista Tecnologia e Ambiente, v. 21, 2014, Criciúma, Santa Catarina. ISSN: 1413-8131 (versão impressa) ISSN: 2358-9426 (versão eletrônica)

Pode-se verificar ainda na área reabilitada a ausência de algumas espécies predadoras generalistas que ocorreram na área do remanescente, pertencentes aos gêneros Eciton, Ectatomma, Gnamptogenys, Heteroponera, Neoponera e Odontomachus, que possivelmente está relacionada à reduzida disponibilidade de presas. No entanto, é provável também que as espécies pertencentes a estes gêneros sejam mais adaptadas às condições florestais, o que impede sua recolonização em áreas mais expostas, com condições microclimáticas bem diferentes daquelas no interior da floresta. Segundo Schmidt et al. (2013), a reduzida cobertura vegetal em áreas alteradas pode afetar diferentes grupos de forma diferenciada, sendo de forma negativa para aquelas espécies especialistas em áreas fechadas e mais frias, e positivamente aquelas especializadas em áreas mais abertas e mais quentes. Espécies pertencentes ao gênero Eciton, por exemplo, dependem de condições específicas de sombreamento, umidade e temperatura para sobreviver (LAPOLA; FOWLER, 2008). As espécies deste gênero também requerem microhabitats que somente a serapilheira de florestas nativas pode prover para a manutenção de seus ninhos e forrageio (FOWLER et al., 1991; GOTWALD, 1995; LAPOLA; FOWLER, 2008). Segundo Lattke (2003), espécies do gênero Gnamptogenys são comumente encontradas em florestas úmidas, nidificando em troncos caídos, no solo ou serapilheira. Neste sentido, portanto, é possível que a dissimilaridade entre as áreas estudadas seja reduzida com um tempo maior de recuperação da área anteriormente minerada, desde que haja uma melhoria nas condições físicas do solo, além de um incremento na densidade e na riqueza de espécies arbóreas e de melhorias nas condições do solo. Além disso, é provável que a riqueza de espécies de formigas aumente com o tempo de reabilitação (ANDERSEN et al., 2004; MAJER et al., 1984). No entanto, a constatação deste processo de regeneração requer um prazo mais longo de monitoramento.

Logo, o presente estudo mostra que o processo de reabilitação das áreas não ofereceu até o momento as condições necessárias para a manutenção de uma riqueza elevada de espécies de formigas, assim como uma composição de espécies mais próxima das condições originais, devido, possivelmente, a reduzida disponibilidade de recursos e, talvez, ao aumento da competição interespecífica. Entretanto, mais estudos necessitam ser realizados ao longo do processo de recuperação das áreas, para um melhor entendimento sobre o avanço no grau de recuperação após o processo de 
Revista Tecnologia e Ambiente, v. 21, 2014, Criciúma, Santa Catarina. ISSN: 1413-8131 (versão impressa) ISSN: 2358-9426 (versão eletrônica)

reabilitação, evidenciando-se, neste caso, a importância do uso de formigas como organismos bioindicadores. Cabe ressaltar, ainda, que tais estudos permitirão não somente avaliar as condições do ambiente, mas possibilitarão também propor alternativas de manejo necessárias para um melhor processo de recuperação. Além disso, o presente estudo evidencia a importância dos remanescentes florestais para a preservação da biodiversidade nativa e para a redução dos impactos ocasionados pela mineração.

Agradecimentos: Os autores agradecem ao Museu de Zoologia da Universidade de São Paulo (USP) e ao Rodolfo da Silva Probst pela confirmação e identificação das espécies coletadas neste estudo. Agradecem à Universidade do Extremo Sul Catarinense (UNESC) pela bolsa de Iniciação Científica concedida ao segundo autor. Agradecem, ainda, ao Sindicato das Indústrias de Extração de Carvão do Estado de Santa Catarina SIECESC, pela autorização da realização da pesquisa nas suas propriedades.

\section{REFERENCIAS}

AGOSTI, D.; MAJER, J. D.; ALONSO, L. E.; SCHUlTZ, R. Ants: Standard Methods for Measuring and Monitoring Biodiversity. Washington: Smithsonian Institution Press, 2000. 280p.

ANDERSEN, A. N.; FISHER, A.; HOFFMANN, B. D.; READ, J. L.; RICHARDS, R. Use of terrestrial invertebrates for biodiversity monitoring in Australian rangelands, with particular reference to ants. Austral Ecology, v. 29, n. 1, p. 87-92, 2004.

ANDRADE, T.; MARQUES, G. D. V.; DEL-CLARO, K. Diversity of ground dwelling ants in Cerrado: na anlysis of temporal variations and distinctive physiognomies of vegetation (Hymenoptera: Formicidae). Sociobiology, v. 50, p. 1-14, 2007.

ANEEL - Agência Nacional de Energia Elétrica. 2008. Atlas de energia elétrica do Brasil: O processo de energia elétrica a partir do carvão mineral. Brasilia, $3^{\circ}$ edição. Disponível em: 〈http://www.aneel.gov.br/>. Acesso em: 02 julho 2014.

BELCHIOR, C.; DEL-CLARO, K.; OLIVEIRA, P. S. Seasonal patterns in the foraging ecology of the harvester ant Pogonomyrmex naegelii (Formicidae, Myrmicinae) in a Neotropical savanna: daily rhythms, shifts in granivory and carnivory, and home range. Arthropod-Plant Interactions, v. 6, p. 571-582, 2012.

BELOLLI, M. A história do carvão de Santa Catarina. Florianópolis: Imprensa Oficial do Estado de Santa Catarina. 2002. 296p.

BRANDÃO, C. R. F.; SILVA, R. R.; DELABIE, J. C. Neotropical ants (Hymenoptera) functional groups: nutritional and applied implications. In: PANIZZI; A. R; PANIZZI, 
Revista Tecnologia e Ambiente, v. 21, 2014, Criciúma, Santa Catarina. ISSN: 1413-8131 (versão impressa) ISSN: 2358-9426 (versão eletrônica)

J. R. P. (eds.): Insect bioecology and nutrition for integrated pest management. Boca Raton: CRS Press. 2012. 213-236 p.

CAMPOS, M. L.; ALMEIDA, J. A.; SOUZA, L. S. Avaliação de três áreas de solo construído após mineração de carvão a céu aberto em Lauro Müller, Santa Catarina. Revista Brasileira de Ciência do Solo, v. 27, p. 1123-1137, 2003.

CAMPOS, M. L.; ALMEIDA, J. A.; SILVEIRA, C. B.; GATIBONI, L. C.; ALBUQUERQUE, J. A.; MAFRA, A. L.; MIQUELLUTI, D. J.; KLAUBERG, O.; SANTOS, J. C. P. Impactos no solo provocados pela mineração e depósito de rejeitos de carvão mineral. Revista de Ciências Agroveterinárias, v. 9, n. 2, p. 198-205, 2010.

CARROL, C. R.; RISCH, S. J. The dynamics of seed harvesting in early successional communities by a tropical ant, Solenopsis geminate. Oecologia, v. 61, p. 388-392, 1984.

CHACÓN DE ULLOA, P. Hormigas urbanas. In: FERNÁNDEZ, F. (ed.): Introducción a las hormigas de la región neotropical. Colômbia: Instituto de Investigación de Recursos Biológicos Alexander Von Humbolt. 2003. p. 351-359.

CITADINI-ZANETTE, V.; SANTOS, R.; KLEIN, A. S.; MARTINS, R.; BRUMFIGUEIRÓ, A. C. Vegetação arbustivo-arbórea em fragmentos florestais do sul de Santa Catarina, Brasil. In: MILIOLI, G.; SANTOS, R.; CITADINI-ZANETTE, V. (eds.): Mineração de carvão, meio ambiente e desenvolvimento sustentável no sul de Santa Catarina: Uma abordagem interdisciplinar. Curitiba: Juruá, 2009. 316 p.

COELHO, M. S.; FERNANDES, G. W. SANTOS, J. C.; DELABIE, J. H. C. Ants (Hymenoptera: Formicidae) as bioindicators of land restoration in a Brazilian atlantic forest fragment. Sociobiology, v. 54, n. 1, p. 51-63, 2009.

CORRÊA, M. M.; FERNANDES, W. D.; LEAL, I. R. Diversidade de formigas epigéicas (Hymenoptera: Formicidae) em capões do Pantanal Sul Matogrossense: relações entre riqueza de espécies e complexidade estrutura da área. Neotropical Entomology, v. 35, p. 724-730, 2006.

COSTA, C. B.; RIBEIRO, S. P.; CASTRO, P. T. A. Ants as bioindicators of natural succession in savanna and riparian vegetation impacted by dredging in the Jequitinhonha River Basin, Brazil. Restoration Ecology, v. 18, n. 1, p. 148-157, 2010.

COSTA, S.; ZOCCHE, J. J. Fertilidade de solos construídos em áreas de mineração de carvão na região Sul de Santa Catarina. Revista Árvore, v. 33, n. 4, p. 665-674, 2009.

DELABIE, J. H. C.; AGOSTI, D.; NASCIMENTO, I. C. D. Litter ant communities of the Brazilian Atlantic rain forest region. In: MAJER, J. D.; AGOSTI, D.; ALONSO, L.; SCHULTZ, T. (eds). Sampling ground-dwelling ants: case studies from the worlds' rain forests. School of Environmental Biology, Perth, Western, Australia, 2000. p. 117 .

DELABIE, J. H. C.; FOWLER, H. G. Soil and litter cryptic ant assemblages of Bahia cocoa plantations. Pedobiologia, v. 39, n. 1 p. 423-433, 1995.

DELABIE, J. H. C.; PAIM, V. R. L. D.; NASCIMENTO, I. C. D.; CAMPIOLO, S.; MARIANO, C. D. S. F. As formigas como indicadores biológicos do impacto humano em manguezais da costa sudeste da Bahia. Neotropical Entomology, v. 35, p. 602-615, 2006. 
DE LUCA, F. J.; GASTALDON, M. C. Desenvolvimento sustentável e a recuperação das áreas degradadas abandonadas pela mineração de carvão na região Sul do Estado de Santa Catarina. Revista tecnologia e Ambiente, v. 5, n. 2, p. 19-33, 1999.

DIAS, L. E.; GRIFFTITH, J. J. Conceituação e caracterização de áreas degradadas. In: DIAS, L. E.; MELlO, J. W. V. (eds.): Recuperação de Áreas Degradadas. Universidade Federal de Viçosa, Sociedade Brasileira de Recuperação de Áreas Degradadas. 1998. 251p.

FERNÁNDEZ, F. Subfamilia Myrmicinae. In: FERNÁNDEZ, F. (ed.): Introducción a las hormigas de la región neotropical. Colômbia: Instituto de Investigación de Recursos Biológicos Alexander Von Humbolt, 2003. p. 307-330.

FOWLER, H. G.; BUENO, O. C.; SADATSUNE, T.; MONTElli, A. C. Ants as potential vectors of pathogens in hospitals in the state of São Paulo, Brazil. Insect Science Application, v. 14, n. 3, p. 367-370, 1993.

FOWLER, H. G.; FORTI, L. C.; BRANDÃO, C. R. F.; DELABIE, J. H. C.; VASCONCELOS, H. L. Ecologia nutricional de formigas. In: Panizzi, A.R.; Parra, J.R.P. (ed). Ecologia nutricional de insetos e suas aplicações no manejo de pragas. São Paulo: Editora Manole, 1991. p. 131-223.

FOWLER, H. G.; SCHLINDWEIN, M. N.; MEDEIROS, M. A. Exotic ants and community simplification in Brazil: A review of the impact of exotic ants on native ant assemblages. In: Williams (ed.). Exotics ants: Biology, Impact and Control of Introduced Species. Westview: Press, Boulder, 1994. p.151-162.

GOLLAN, J. R.; BRUYN, L. L. D.; REID, N.; SMITH, D.; WILKIE, L. Can ants be used as ecological indicators or restoration progress in dynamic environments? A case study in a revegeted riparian zone. Ecological Indicators, v. 11, n. 6, p. 1517-1525, 2011.

GONÇALVES, C. R.; NUNES, A. M. Formigas das praias e restingas do Brasil. In: LACERDA, L. D.; ARAÚJO, D. S. D.; CERQUEIRA, R.; TUREQ, B. (eds). Restingas: origem, estrutura, processos. Niterói: CEUFF, 1994. p. 373-378.

GOTWALD, J. R. Army ants. New York: Cornell University Press, 1995. 302p.

HÖLLDOBLER, B.; WILSON, E. O. The ants. Cambridge: Harvard University Press, 1999. p. 897-898.

HOLLWAY, D. A.; LACH, L.; SUAREZ, A. V.; TSUITSUI, N. D.; CASE, T. J. The causes and consequences of ant invasions. Annual Review of Ecology and Systematics, v. 33, p. 181-233, 2002.

IPAT - Instituto de Pesquisas Ambientais e Tecnológicas. Projeto técnico de recuperação de áreas degradadas: Lotes 61. Universidade do Extremo Sul Catarinense, Criciúma. 2000. 79 p.

JORDAN, D.; KREMER, R. J.; BERGFIELD, W. A.; KIM, K. Y.; CACNIO, V. N. Microbial and faunal biomass in soils. Biology and Fertility of Soils, v. 19, p. 297-302, 1995.

KLEIN, A. S.; CITADINI-ZANETTE, V.; SANTOS, R.; PEREIRA, J. L. Florística, aspectos ecológicos e reprodutivos da comunidade vascular em área degradada pela mineração de carvão. In: MILIOLI, G.; SANTOS, R.; CITADINI-ZANETTE, V. (eds.): 
Revista Tecnologia e Ambiente, v. 21, 2014, Criciúma, Santa Catarina. ISSN: 1413-8131 (versão impressa) ISSN: 2358-9426 (versão eletrônica)

Mineração de carvão, meio ambiente e desenvolvimento sustentável no sul de Santa Catarina: Uma abordagem interdisciplinar. Curitiba: Juruá, 2009. 316 p.

KÖPPEN, W. Climatologia: com um estúdio de los climas de la tierra. Fondo de cultura Económica, México. 1948.

LAPOLA, D. M.; FOWLER, H. G. Questioning the implementation of habitats corridors: a case study in interior São Paulo using ants as bioindicators. Brazilian Journal Biology, v. 68, n. 1, p. 11-20, 2008.

LASSAU, S.A.; HOCHULI, D.F. Effects of habitat complexity on ant assemblages. Ecography, v.27, p. 157-164, 2004.

LATTKE, J. E. Subfamilia Ponerinae. In: FERNÁNDEZ, F. (ed.): Introducción a las hormigas de la región neotropical. Colômbia: Instituto de Investigación de Recursos Biológicos Alexander Von Humbolt, 2003. p. 261-276.

LEAL, I. L.; OLIVEIRA, P. S. Interaction between fungus-growing ants (Attini), fruits and seeds in Cerrado vegetation in southes Brazil. Biotropica, v. 30, n. 2, p. 170-178, 1998.

LINDENMAYER, D. B. Future directions for biodiversity conservation in managed forests: indicator species, impact studies and monitoring programs. Forest Ecology and Management, v. 115, p. 277-287, 1999.

LOPES, D. T.; LOPES, J.; NASCIMENTO, I. C. D.; DELABIE, J. H. C. Diversidade de formigas epigéicas no Parque Estadual Mata do Godoy, Londrina, Paraná. Iheringia, v. 100, p. 84-90, 2010.

LOBRY DE BRUYN, A. Ants as bioindicators of soil function in rural environments. Agriculture and Environment, v. 74, n. 1-3, p. 425-441, 1999.

LUTINSKI, J. A.; LOPES, B. C.; MORAIS, A. B. B. Diversidade de formigas urbanas (Hymenoptera: Formicidae) de dez cidades do sul do Brasil. Biota Neotropica, v.13, n.3, p. 333-342, 2013.

MAJER, J. D. Ants: bio-indicators of minesite rehabilitation, landuse and land conservation. Environmental Management, v. 7, p. 375-383, 1983.

MAJER, J. D.; BRENNAN, K. E. C.; MOIR, M. L. Invertebrates and the restoration of a forest ecosystem: 30 years of research following bauxite mining in Western Australia. Restoration Ecology, v. 15, n. 4, p. 104-115, 2007.

MAJER, J. D.; DAY, J. E.; KABAY, E. D.; PERRIMAN, W. S. Recolonization by ants bauxite mines rehabilited by a number of different methods. Journal of Applied Ecology, v. 21, p. 355-375, 1984.

MMA - Ministério do Meio Ambiente. 2011. Monitoramento do desmatamento nos biomas brasileiros por satélite. Disponível em: <http://www.mma.gov.br.> Acesso em: 01 maio 2014.

MARINHO, C. G. S.; ZANETTI, R.; DELABIE, J. H. C.; SCHLINDWEIN, M. N.; RAMOS, L. S. Diversidade de formigas (Hymneoptera: Formicidae) da serapilheira em eucaliptais (Myrtaceae) e área de cerrado de Minas Gerais. Neotropical Entomology, v. 31, p. 187-195, 2002. 
OTTONETTI, L.; TUCCI, L.; SANTINI, G. Recolonization patterns of ants in a rehabilitated lignite mine in Central Italy: potential for the use of Mediterranean ants as indicators of restoration processes. Restoration Ecology, v. 14, n. 1, p. 60-66, 2006.

PALACIO, E. E.; FERNÁNDEZ, F. Claves para las subfamílias y gêneros. In: FERNÁNDEZ, F. (ed.): Introducción a las hormigas de la región neotropical. Colômbia: Instituto de Investigación de Recursos Biológicos Alexander Von Humbolt, 2003. p. 233-260.

PASSERA, L. Quels sont les caracteres ethophysiologiques des "fourmis vagabondes"?. Actes Colloque Insectes Sociaux, v. 8, p. 39-45, 1993.

PASSERA, L. Charateristics of tramp Species. In: WILLIAMS, D. F. (ed.). Exotics ants: Biology, Impact and Control of Introduced Species. Boulder: Wesrview Press, 1994. p. 23-43.

PECK, S. I.; MCQUAID, B.; CAMPBELL, C. L. Using ant species as a biological indicator of agroecosystems condition. Environmental Entomology, v. 27, p. 11021110, 1998.

PEREIRA, M. P.; QUEIROZ, J.; VALCARCEL, R.; MAYHÉ-NUNES, A. J. Fauna de formigas como ferramenta para monitoramento de área de mineração reabilitada na Ilha da Madeira, Itaguaí, RJ. Ciência Florestal, v. 17, p. 197-204, 2007.

PHILPOTT, S. M.; PERFECTO, I.; ARMBRECHT, I.; PARR, C. L. Ant diversity and function in disturbed and changing habitats. In: LACH, L.; PARR, C. L.; ABBOTT, K. L. (eds.): Ant Ecology. Oxford: Oxford University Press, 2010. p. 137-156.

PINTO, L. F. S. Potencial de acidificação e de neutralização dos materiais geológicos para a composição do solo construído em áreas de mineração de carvão. 1997. Tese (Doutorado) - Universidade Federal do Rio Grande do Sul, Porto Alegre. 1997.

QUEIROZ, J. M.; ALMEIDA, F. S.; PEREIRA, M. P. D. S. Conservação da biodiversidade $\mathrm{e}$ o papel das formigas (Hymenoptera: Formicidae) em agroecossistemas. Floresta e Ambiente, v. 13, p. 37-45, 2006.

RAMOS, L. S.; MARINHO, C. G. S.; FILHO, R. Z. B.; DELABIE, J. H. C.; LACAU, S.; SANTOS, M. D. F. S. D.; NASCIMENTO, I. C. D. Comunidades de formigas (Hymenoptera: Formicidae) de serapilheira em áreas de cerrado "stricto sensu" em Minas Gerais. Lundiana, v. 4, p. 95-102, 2003.

RIBAS, C. R.; CAMPOS, R. B. F.; SCHMIDT, F. A.; SOLAR, R. R. C. Ants as indicators in Brazil: a review with suggestions to improve the use of ants in environmental monitoring programs. Psyche, p. 1-23, $2012 \mathrm{a}$.

RIBAS, C. R.; SCHMIDT, F. A.; SOLAR, R. R. C.; CAMPOS, R. B. F.; VALENTIM, C. L.; SCHOEREDER, J. H. Ants as indicators of the success of rehabilitation efforts in deposits of gold mining tailings. Restoration Ecology, v. 20, n. 6, p. 712-720, 2012 b.

RIBAS, C.R.; SCHOEREDER, J.H.; PIC, M.; SOARES, S.M. Tree heterogeneity resource availability, and larger scale process regulating arboreal ant species richness. Austral Ecology, v. 28, p. 305-314, 2003. 
RIBAS, C. R.; SOLAR, R. R. C.; CAMPOS, R. B. F.; SCHMIDT, F. A.; VALENTIM, C. L.; SCHOEREDER, J. H. Can ants be used as indicators of environmental impacts caused by arsenic? Journal of Insect Conservation, v. 16, n. 3, p. 413-421, 2012c.

RISCH, S. J.; CARROL, C. R. The ecological role of ants in two Mexican agroecosystems. Oecologia, v. 55, p. 114-119, 1982.

ROCHA-NICOLEITE, E.; CAMPOS, M.L.;CITADINI-ZANETTE, V.; SANTOS, R.; MARTINS, R.; SOARES, C.R.F.S. 2013. Mata Ciliar: Implicações técnicas sobre a restauração após mineração de carvão. Criciúma, SATC. 2013. 80p.

ROSADO, J. L. O.; GONÇALVES, M. G.; DRÖSE, W.; SILVA, E. J. E.; KRÜGER, R. F.; FEITOSA, R. M.; LOECK, A. E. Epigeic ants (Hymenoptera: Formicidae) in vineyards and grassland areas in the Campanha region, state of Rio Grande do Sul, Brazil. Check List, v. 8, n. 6, p. 1184-1189, 2012.

SANCHES, J. C. D.; FORMOSO, M. L. L. Utilização do carvão e meio ambiente. Porto Alegre: CIENTEC, 1990. 34p.

SCHMIDT, F. A.; DIEHL, E. What is the effect of soil use on ant communities? Neotropical Entomology, v. 37, p. 381-388, 2008.

SCHMIDT, F. A.; RIBAS, C. R.; SCHOEREDER, J. H. How predictable is the response of ant assemblages to natural forest recovery? Implications for their use as bioindicators. Ecological Indicators, v. 24, p. 158-166, 2013.

SILVA, R. R.; BRANDÃO, C. R. F. Formigas (Hymenoptera: Formicidae) como indicadores da qualidade ambiental e da biodiversidade de outros invertebrados terrestres. Biotemas, v.12, n.2, p. 55-73, 1999.

SILVESTRE, R. C.; BRANDÃO, R. F.; SILVA, R. R. Grupos funcionales de hormigas: el caso de los grêmios del Cerrado. In: FERNÁNDEZ, F. (ed.): Introducción a las hormigas de la región neotropical. Colômbia: Instituto de Investigación de Recursos Biológicos Alexander Von Humbolt, 2003. p. 113-148.

TSUTSUI, N. D.; SUAREZ, A. V. The colony structure and population biology of invasive ants. Conservation Biology, v. 17, n. 1, p. 48-58, 2003.

UNDERWOOD, E.C.; FISHER, B.L. 2006. The role of ants in conservation monitoring: if, when, and how. Biological Conservation, v. 132, n. 2, p. 166-182, 2006.

VASCONCELOS, H. L. Effects of forest disturbance on the strucutre of groundforanging ant communities in central Amazonia. Biodiversity and Conservation, v. 8, p. 409-420, 1999.

VASCONCELOS, H.L.; LEITE, M.F.; VILHENA, J.M.S.; LIMA, A.P.; MAGNUSSON, W.E. Ant diversity in na Amazonian savana: relationship with vegetation structure, disturbance by fire, and dominant ants. Austral Ecology, v.33, p. 221-231, 2008.

VASCONCELOS, H. L.; VILHENA, J. M. S.; CALIRI, G. J. A. Responses of ants to selective logging of a central Amazonian forest. Journal of Applied Ecology, v. 37, n. 3, p. 508-514, 2000. 
Revista Tecnologia e Ambiente, v. 21, 2014, Criciúma, Santa Catarina. ISSN: 1413-8131 (versão impressa) ISSN: 2358-9426 (versão eletrônica)

VASCONCELOS, H.L.; VILHENA, J.M.S.; FACURE, K.G.; ALBERNAZ, A.L.K.M. Patterns of ant species diversity and turnover across 2000km of Amazonian floodplain Forest. Journal of Biogeography, v. 37, n.3, p.432-440, 2010.

WILSON, E. O. Pheidole in the new world: a dominant, hyperdiverse ant genus. Harvard University Press, Cambridge. 2003. 818p. 